UCRL-JC- 119497

\title{
PREPRINT
}

\section{Adaptive Ocean Acoustic Processing for a Shallow Ocean Experiment}

\author{
J. V. Candy \\ Lawrence Livermore National Laborator \\ E. J. Sullivan \\ Naval Undersea Warfare Center
}

This paper was prepared for submission to the

OCEANS '95 MTS/IEEE

October 9-12, 1995, San Diego, CA

July 19, 1995

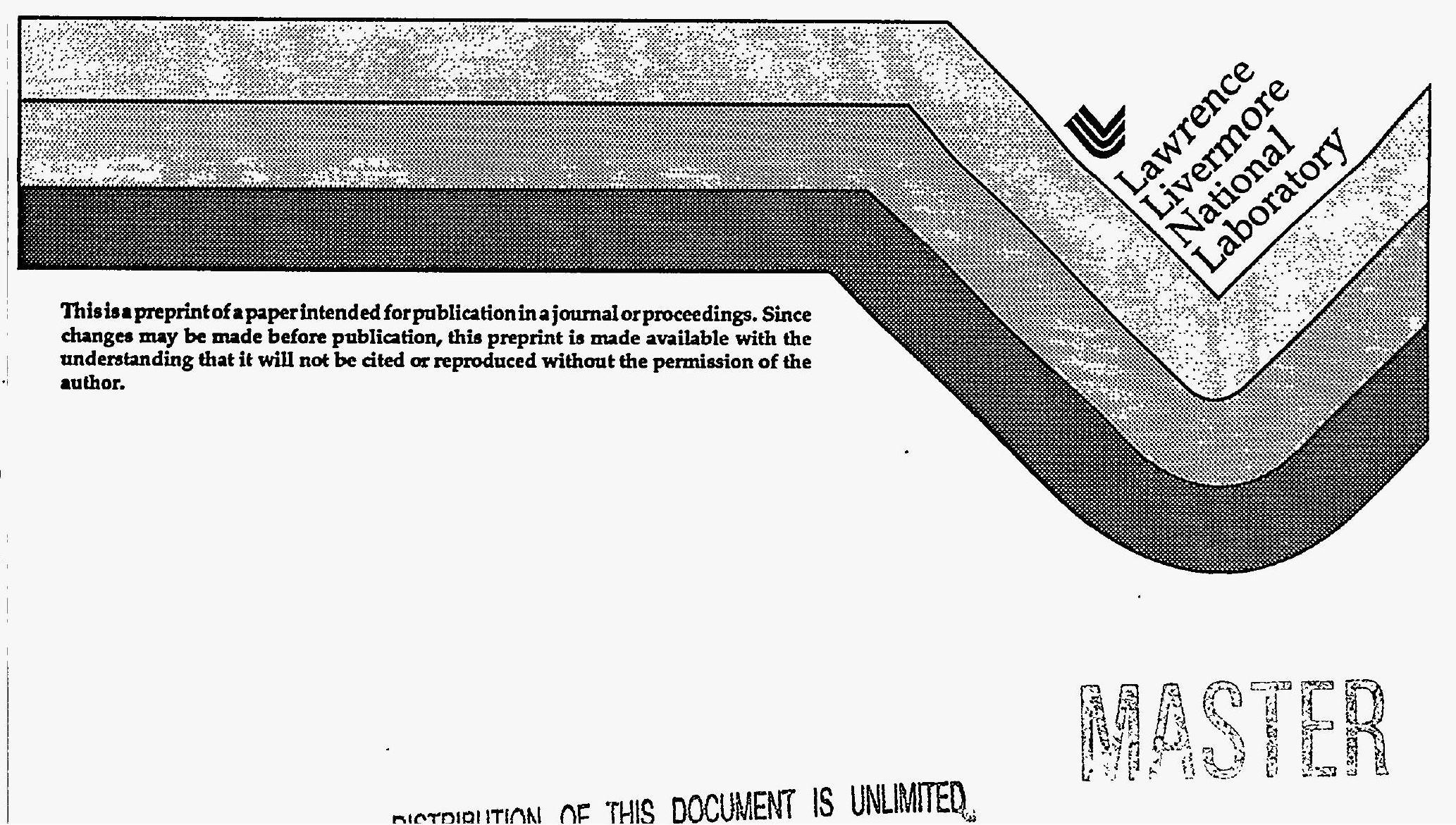




\section{DISCLAIMER}

This document was prepared as an account of work sponsored by an agency of the United States Government. Neither the United States Government nor the University of California nor any of their employees, makes any warranty, express or implied, or assumes any legal liability or responsibility for the accuracy, completeness, or usefulness of any information, apparatus, product, or process disclosed, or represents that its use would not infringe privately owned rights. Reference herein to any specific commercial product, process, or service by trade name, trademark, manufacturer, or otherwise, does not necessarily constitute or imply its endorsement, recommendation, or favoring by the United States Government or the University of California. The views and opinions of authors expressed herein do not necessarily state or reflect those of the United States Government or the University of California, and shall not be used for advertising or product endorsement purposes. 


\section{DISCLAIMER}

Portions of this document may be illegible in electronic image products. Images are produced from the best available original document. 


\section{Adaptive Ocean Acoustic Processing for a Shallow Ocean Experiment}

\author{
J.V. Candy \\ Lawrence Livermore National Laboratory \\ Livermore, CA 94550 \\ E.J. Sullivan \\ Naval Undersea Warfare Center \\ Newport, RI 02841
}

\begin{abstract}
A model-based approach is developed to solve an adaptive ocean acoustic signal processing problem. Here we investigate the design of a model-based identifier (MBID) for a normal-mode model developed from a shallow waterr ocean experiment and then apply it to a set of experimental data demonstrating the feasibility of this approach. In this problem we show how the processor can be structured to estimate the horizontal wave numbers directly from measured pressurefield and sound speed thereby eliminating the need for synthetic aperture processing or a propagation model solution.
\end{abstract}

\section{INTRODUCTION}

Ocean acoustic signal processing has made great strides over the past decade necessitated by the development of quieter submarines and the recent prolifieration of diesel powered vessels. These improvements have been achieved by developing processors that incorporate knowledge of the surrounding ocean environment and noise into their processing schemes [1-4]. However, it is wellknown that if the incorporated model is inaccurate either parametrically or incorrect from the basic principles, then the processor can actually perform worse in the sense that the predicted error variance is greater than that of the raw measurements $[5,6]$. In fact, one way to choose the "best" model or processor is based on comparing predicted error variances - the processor achieving the smallest wins! In practice, the usual procedure to check for model adequacy is to analyze the statistical properties of the resulting residual or innovations sequence, that is, the difference between the measured and predicted measurements. Here again the principle of minimum (residual) variance is applied to decide on the best processor or equivalently the best embedded model [7]. Other sophisticated statistical tests have been developed for certain classes of models with high success to make this decision [8-10]. In any case the major problem with modelbased signal processing (MBP) schemes is assuring that the model incorporated in the algorithm is ad- equate for the proposed application, that is, it can faithfully represent the on-going phenomenology. Therefore, it is necessary, as part of the MBP design procedure, to estimate/update the model parameters either through separate experiments or jointly (adaptively) while performing the required processing $[11,12]$. The introduction of a recursive, on-line MBP can offer a dramatic detection improvement in a tactical passive or active sonartype system especially when a rapid environmental assessment is required $[12,13]$. In this paper, we discuss the development of a processor capable of adapting to the ever changing ocean environment and providing the required signal enhancement-for detection and localization.

Here we investigate the development of a "model-based identifier," (MBID) that is, a identifier that incorporates an initial mathematical representation of the ocean acoustic propagation model into its framework and adapts, on-line, its parameters as the ocean changes environmentally. Here we are interested primarily in a shallow water environment characterized by a normal-mode model and therefore, our development will concentrate on adaptively adjusting parameters of the normal-mode propagation model to "fit" the ocean surrounding our sensor array. In fact, one way to think about this processor is that it passively listens to the ocean environment and "learns" or adapts to its changes. It is clear that the resulting processor will be much more sensitive to changes than one that does not, thereby, providing current information and processing. The algorithm uses the incoming data to adaptively update the parameter set, jointly, with the acoustic signal processing. In the following, we define the MBID as a Kalman filter whose estimated states are the modal functions $\hat{\phi}\left(z_{\ell}\right)$ and states representing the estimated ocean acoustic parameters $\hat{\theta}\left(z_{l}\right)$ that have been augmented into the processor. The basic processor is shown in Figure 1. The inputs to the MBID are raw data $\left[\left\{p\left(z_{\ell}\right)\right\},\left\{c\left(z_{\ell}\right)\right\}\right]$ 


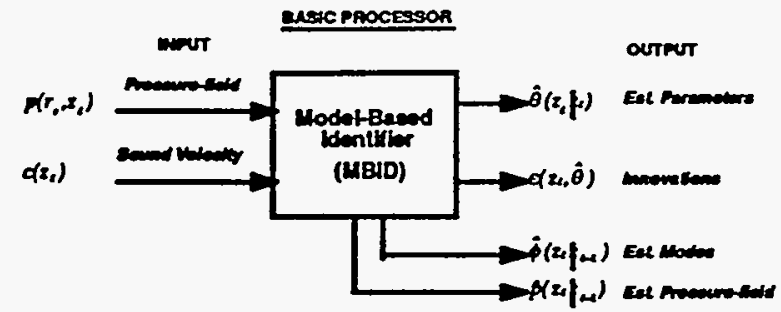

Figure 1: Model-Based Ocean Acoustic Identification: the Basic Processor.

and its outputs are $\theta\left(z_{\ell}\right)$ the set of parameters of interest.

The application of this adaptive approach to other related problems of interest is apparent. For signal enhancement, the adaptive MBP or MBID can provide enhanced signal estimates of modal functions (modal filtering), pressure-field estimates (measurement filtering), and parameters of interest (parameter estimation) such as wave numbers, range-depth functions, sound speed, etc. $[11,12]$. For model monitoring and source detection purposes, the MBID provides estimates of the residuals or innovations sequence, which can be statistically tested for adequacy [8] or used to calculate a decision function [11]. For localization, the MBID provides estimates of the enhanced range-depth and modal (modal filter) functions used for model-based localization as discussed in a recent paper [14]. In fact for rapid assessment of the ocean environment $-a$ definite requirement in a tactical situation - it is possible to perform madel-based inversion, on-line, using the MBID scheme to adaptively estimate the changing parameters characterizing the sound speed profile $[12,13]$. Thus, the MBID provides a technique capable of "listening and learning".

\section{ADAPTIVE WAVE NUMBER ESTI- MATION}

In this section we develop a model-based identifier for use with an ocean acoustic propagation model. System identification is typically concerned with the estimation of a model and its associated parameters from noisy measurement data. Usually the model structure is pre-defined (as in our case) and then a parameter estimator is developed to "fit" parameters according to some error criterion. After completion or during this estima- tion, the quality of the estimates must be evaluated to decide if the processor performance is satisfactory or equivalently the model adequately represents the data. There are various types (criteria) of identifiers employing many different model (usually linear) structures [8-10]. Since our efforts are primarily aimed ocean acoustics in which the models and parameters are usually nonlinear, we will concentrate on developing a parameter estimator capable of on-line (shipboard) operations and nonlinear dynamics.

From our previous work, it is clear the the extended Kalman filter (EKF) identifier will satisfy these constraints nicely. The general nonlinear identifier or equivalently parameter estimator structure can be derived directly from the EKF algorithm (see Refr. [16] for details). We note that this algorithm is not implemented in this fashion, it is implemented in the numerically stable UDfactorized form as in SSPACK_PC [18].

For propagation in a shallow water environment we choose the normal-mode model which can easily be placed in state-space form (see Refr. 11 for details). We choose the "depth only" structure and assume a vertical array which yields a linear space-varying formulation, then we develop the identifier - a nonlinear processor.

Assuming a horizontally-stratified ocean of depth $h$ with a known source position $(x, y)$ and also that the acoustic energy from a point source can be modeled as a trapped wave governed by the wave equation. We follow the approach of Clay [15] using the separation of variables technique leading to a set of ordinary differential equations. The resulting depth relation is an eigenvalue equation in $z$ with

$$
\frac{d^{2}}{d z^{2}} \phi_{m}(z)+\kappa_{z}^{2}(m) \phi_{m}(z)=0, m=1, \cdots, M
$$

whose eigensolutions $\left\{\phi_{m}(z)\right\}$ are the so called modal functions and $\kappa_{z}$ is the wavenumber in the z-direction. These solutions depend on the sound speed profile, $c(z)$, and the boundary conditions at the surface and bottom as well as the corresponding dispersion relation given by

$$
\kappa^{2}=\frac{\omega^{2}}{c^{2}(z)}=\kappa_{r}^{2}(m)+\kappa_{z}^{2}(m), \quad m=1, \ldots, M
$$

where $\kappa_{r}, \kappa_{z}$ are the respective wave numbers in the $r$ and $z$ directions with $c$ the depth-dependent sound speed profile and $\omega$ the harmonic source frequency.

For our purpose we are concerned with the estimation of the pressure field, therefore we remove 
the time dependence, normalize units, and obtain the acoustic pressure propagation model,

$$
p(r, z)=\sum_{m=1}^{M} q \phi_{m}\left(z_{s}\right) \phi_{m}(z) \frac{e^{-\alpha_{r}(m) r}}{\sqrt{\kappa_{r}(m) r}} e^{j \kappa_{\tau}(m) r}
$$

where $p$ is the acoustic pressure; $q$ is the source amplitude; $\phi_{m}$ is the $m^{t h}$ modal function at $z$ and $z_{s} ; \alpha$ is the modal attenuation; $\kappa_{r}(m)$ is the horizontal wavenumber associated with the $m^{\text {th }}$ mode; and $r$ is the horizontal range.

The normal-mode solutions can easily be placed in state-space form and we refer the interested reader to Refs. 12 and 13 for the detailed theory. This approach leads to a Gauss-Markov representation which includes the second order statistics. The measurement noise can represent the nearfield acoustic noise field, flow noise on the hydrophone and electronic noise. The modal noise can represent sound speed errors, distant shipping noise, errors in the boundary conditions, sea state effects and ocean inhomogeneities. By assuming that the horizontal range of the source $r_{\varepsilon}$ is known a-priori we can use the Hankel function $H_{0}\left(\kappa_{r} r_{a}\right)$ which is the source range solution; therefore, we reduce the state-space model to that of "depth only" and the Gauss-Markov model for this constrained problem is given by

$$
\frac{d}{d z} \phi(z)=A(z) \phi(z)+B(z) u(z)+w_{\phi}(z)
$$

where

$$
A_{m}(z)=\left[\begin{array}{cc}
0 & 1 \\
-\kappa_{z}^{2}(m) & 0
\end{array}\right]
$$

with the pressure field measurement model given by

$$
p\left(r_{a}, z\right)=\mathbf{C}^{T}\left(r_{a}, z\right) \phi(z)+\mathbf{v}(z)
$$

where

$C^{T}\left(r_{s}, z\right)=\left[\beta_{1}\left(r_{s}, z_{s}\right) \quad 0\left|\beta_{2}\left(r_{s}, z_{s}\right) \quad 0\right| \cdots \mid \beta_{M}\left(r_{s}, z_{s}\right) \quad 0\right]$

with $\beta_{m}\left(r_{a}, z_{a}\right)=\frac{g \phi_{m}\left(x_{a}\right)}{\int_{0}^{h} \phi_{m}^{2}(z) d z} H_{0}\left(\dot{k}_{\tau}(m) r_{c}\right)$. The random noise vectors $w_{\phi}$ and $v$ are assumed gaussian, zero-mean with respective covariance matrices, $\boldsymbol{R}_{\boldsymbol{w}_{+} w_{+}}$and $\boldsymbol{R}_{\boldsymbol{v}}$.

Since our array spatially samples the pressurefield, we choose to discretize the differential state equations using a finite (first) difference approach, that is, for the $m^{t h}$-mode we use

$$
\frac{d}{d z} \phi_{m}\left(z_{\ell}\right) \approx \frac{\phi_{m}\left(z_{\ell+1}\right)-\phi_{m}\left(z_{\ell}\right)}{\Delta z_{\ell}}
$$

where $\Delta z_{\ell}=z_{\ell+1}-z_{\ell}$. Therefore, substituting into the differential equation, we obtain

$$
\begin{aligned}
\phi_{m}\left(z_{\ell+1}\right) & =\left[I+\Delta z_{\ell} A_{m}\left(z_{\ell}\right)\right] \phi_{m}\left(z_{\ell}\right) \\
& +\Delta z_{\ell} \mathrm{B}\left(z_{\ell}\right) u\left(z_{\ell}\right)+\Delta z_{\ell} w_{\phi}\left(z_{\ell}\right)
\end{aligned}
$$

Since we have a vertical line sensor array to measure the pressure-field, the measurement model for the $m^{\text {th }}$ mode becomes

$$
\begin{aligned}
p_{m}\left(r_{s}, z_{\ell}\right) & =\mathbf{C}_{m}^{T}\left(r_{s}, z_{\ell}\right) \phi_{m}\left(z_{\ell}\right)+v_{m}\left(z_{\ell}\right) \\
& =\sum_{m=1}^{M} \beta_{m}\left(r_{s}, z_{s}\right) \phi_{m 1}\left(z_{\ell}\right)+v_{m}\left(z_{\ell}\right)
\end{aligned}
$$

It is this model that we employ in our modelbased identifier. Next suppose we assume that the horizontal wave numbers, $\left\{\kappa_{\mathrm{r}}(m)\right\}$, are unknown and we would like to estimate them directly from the pressure-field measurements. Note that the horizontal wave numbers are not a function of depth - they are constant or invariant over depth. This is a crucial first step for any ocean acoustic processing requirement. By estimating the wave numbers directly from the noisy array measurements, not only do we obtain the parameters required for our subsequent model-based processor designs, but also we replace the need to perform the synthetic aperture processing using a towed array which is the usual approach in obtaining the horizontal wave numbers. Once estimated, the horizontal wave numbers along with known sound speed can be used to determine the vertical wave numbers directly from the dispersion relation. Note also that this implies that by identifying the wave numbers directly from the sensor measurements we are essentially eliminating the need to solve the boundary value problem using a numerical eigenvalue solver. This is possible because it is known that the wave numbers carry all of the essential information (boundary, temporal frequency etc.) directly - a powerful set of parameters! Thus, we define the basic wave number identification problem as GIVEN a set of noisy pressure-field and sound speed measurements, $\left[\left\{p\left(r_{s}, z_{\ell}\right)\right\},\left\{c\left(z_{\ell}\right)\right\}\right]$, FIND the "best" (minimum error variance) estimate of the horizontal wave numbers, that is, $\left\{\hat{r}_{r}(m)\right\}, m=1, \cdots, M$.

The basic form of the coupled modal equations follow from Eq. 4 with $\kappa_{r} \longrightarrow \theta$ and $m=1, \cdots, M$

$$
\begin{aligned}
& \phi_{m 1}\left(z_{l}\right)=\phi_{m 1}\left(z_{l-1}\right)+\Delta z_{\ell} \phi_{m 2}\left(z_{l-1}\right) \\
& \phi_{m 2}\left(z_{l}\right)=-\Delta z_{l}\left(\frac{\omega^{2}}{\tau^{2}\left(z_{l-1}\right)}-\theta_{m}^{2}\left(z_{l-1}\right)\right) \phi_{m 1}\left(z_{l-1}\right) \\
& + \\
& \theta_{m}\left(z_{l}\right)=\phi_{m 2}\left(z_{l-1}\right)
\end{aligned}
$$

and with corresponding messurement model

$$
p_{m}\left(r_{s}, z_{l}\right)=\sum_{m=1}^{M} \beta_{m}\left(r_{s}, z_{s}\right) \phi_{m 1}\left(z_{\ell}\right)
$$


The MBID requires the following jacobian matrices for the wave number identification problem [17]

$$
A[\phi, \theta]=\left[\begin{array}{ccc}
A_{\phi}[\phi, \theta] & 1 & A_{\theta}[\phi, \theta] \\
- & - & - \\
0 & 1 & I_{M}
\end{array}\right]
$$

The associated wave number (parameter jacobian) is with $A_{\phi} \in R^{2 M \times 2 M}$, and $A_{\theta} \in R^{2 M \times M}$ and the overall jacobian matrix given by $A[\phi, \theta] \in$ $R^{3 M \times 3 M}$ with

$$
A_{\phi}[\phi, \theta]=A\left(z_{\ell}, \theta\right)
$$

For the measurement system, we have that

$$
c_{m}[\phi, \theta]=\frac{q \phi_{m}\left(z_{s}\right)}{\int_{0}^{h} \phi_{m}^{2}(z) d z} H_{0}\left(k_{r}(m) r_{s}\right) \dot{\phi}_{m 1}\left(z_{\ell}\right)
$$

or substituting for the known and unknown parameters and using the well-known approximation to the Hankel function for $\kappa_{r} r_{a}>1$, we obtain

$c_{m}[\phi, \theta]=\gamma_{m}\left(z_{s}\right) \sqrt{\frac{2}{\pi \theta_{m}\left(z_{\ell}\right) r_{l}}} \cos \left(\theta_{m}\left(z_{\ell}\right) r_{s}-\pi / 4\right) \phi_{m 1}\left(z_{\ell}\right)$

where $\gamma_{m}\left(z_{s}\right):=\frac{q \phi_{m}\left(z_{s}\right)}{\int_{0}^{h} \phi_{m}^{2}(z) d z}$ and $H_{0}\left(\kappa_{r}(m) r_{s}\right) \approx$ $\left(\sqrt{\frac{2}{\pi \theta_{m}\left(x_{l}\right) r_{s}}} \cos \left(\theta_{m}\left(z_{l}\right) r_{s}-\pi / 4\right)\right.$. Summing over the total number of modes we have

$c[\phi, \theta)]=\sum_{m=1}^{M} \gamma_{m}\left(z_{s}\right) \sqrt{\frac{2}{\pi \theta_{m}\left(z_{\ell}\right) r_{s}}} \cos \left(\theta_{m}\left(z_{\ell}\right) r_{s}\right) \phi_{m 1}\left(z_{\ell}\right)$

The $1 \times 3 M$ measurement jacobian vector for this problem is given by

$$
C[\phi, \theta]=\left[C_{\phi}[\phi, \theta] \mid C_{\theta}[\phi, \theta]\right]
$$

Using this information we can easily construct the overall parameter estimator. The prediction equations for the $m^{\text {th }}$ mode and wave number are

$$
\begin{array}{ll}
\hat{\phi}_{m 1}\left(z_{\ell} \mid z_{\ell-1}\right)= & \hat{\phi}_{m 1}\left(z_{\ell-1} \mid z_{\ell-1}\right)+\Delta z_{\ell} \hat{\phi}_{m 2}\left(z_{\ell-1} \mid z_{\ell-1}\right) \\
\hat{\phi}_{m 2}\left(z_{\ell} \mid z_{\ell-1}\right)= & -\Delta z_{\ell}\left(\frac{\omega^{2}}{c^{2}\left(z_{\ell}\right)}-\hat{\theta}_{m}^{2}\left(z_{\ell-1}\right) .\right. \\
& \hat{\phi}_{m 1}\left(z_{\ell-1} \mid z_{\ell-1}\right)+\hat{\phi}_{m 2}\left(z_{\ell-1} \mid z_{\ell-1}\right) \\
\hat{\theta}_{m}\left(z_{\ell} \mid z_{\ell-1}\right)= & \hat{\theta}_{m}\left(z_{\ell-1} \mid z_{\ell-1}\right)
\end{array}
$$

and the corresponding innovations (parameterized by $\theta$ ) are given by

$$
e\left(z_{\ell}, \theta\right)=p\left(r_{s}, z_{\ell}\right)-\sum_{m=1}^{M} c_{m}[\phi, \theta] \hat{\phi}_{m 1}\left(z_{\ell} \mid z_{\ell-1}, \theta\right)
$$

with the correction equations

$$
\begin{aligned}
& \hat{\phi}\left(z_{\ell} \mid z_{\ell}\right)=\hat{\phi}\left(z_{\ell} \mid z_{\ell-1}\right)+K_{\phi}\left(z_{\ell}\right) e\left(z_{\ell}, \theta\right) \\
& \hat{\theta}_{m}\left(z_{\ell} \mid z_{\ell}\right)=\hat{\theta}_{m}\left(z_{\ell} \mid z_{\ell-1}\right)+K_{\theta}\left(z_{\ell}\right) e\left(z_{\ell}, \theta\right)
\end{aligned}
$$

This completes the basic implementation of the MBID, next we discuss its application to a set of noisy experimental measurements. We utilize the experiment at the Hudson Canyon located off of the New Jersey coast, a wellknown shallow water $(73 \mathrm{~m})$ ocean environment [19]. Here a 23-element vertical array is deployed from the bottom with $2.5 \mathrm{~m}$ separation to measure the pressure-field. Note that the subsequent plots show the function over the aperture length $(57.5 \mathrm{~m})$ not its actual position in the water column. We use a $1.25 \mathrm{~m}$ spacing using the following average horizontal wave numbers: $\{0.208,0.199,0.183,0.175,0.142\} m^{-1}$ for the 5 modes supporting the water column from a $36 \mathrm{~m}$ deep, $50 \mathrm{~Hz}$ source at $0.5 \mathrm{Km}$ range (see Refrs. 8 and 33 for more details). We investigate the results of the MBID design using the actual experimental hydrophone measurements from the Hudson Canyon. Here we have the 23 element array and initialize the MBID with the average set of horizontal wave numbers from [18]. The resulting estimates are quite reasonable as shown in Figure 2. Note that although there is a little difficulty tracking the first couple of modes, the results actually appear better than those reported previously for this data set (see Refr. 7). The results for the higher order modes follow those predicted by the model as observed in the figure and corresponding estimation errors. From the figure we see the reconstructed pressure-field and innovations are also quite reasonable as shown in Figure 3 and indicates a "tuned" processor with its zeromean $\left(1.9 \times 10^{-3}<6.7 \times 10^{-3}\right)$ and white. Recall that it is necessary for the innovations sequence to be zero mean and white for the processor to be deemed as "tracking" for the modes and associated parameters. Thus, the processor is successfully tracking and the model is valid for this data set. The final parameter estimates are shown in (19g)ure 4 with the predicted error statistics for this data which are also included in Table I for comparison to the simulated. We note that the parameter estimates continue to adapt to the changing ocean environment based on the pressure-field measurements. We initially start the wave numbers at their averages and then allow them to adapt to the measured sensor data. The first wave number estimate appears to converge (approximately) to the average with a slight bias but the others 
adapt to other values due to changes in the data. We see that the MBID appears to perform better than the fixed MBP (see Table II) with the augmented identifier simply because the horizontal wave numbers are "adaptively" estimated, online providing a superior fit to the raw data. Thus, we see that the use of the MBID in conjunction with vertical array measurements enables us to circumvent the need for a propagation modal solver as long as we have reasonable estimates to initialize the processor. So in this particular application we see how the model-based identifier can be employed to estimate the wave numbers (horizontal) from noisy pressure-field and sound speed measurements evolving using a vertical array or hydrophones. This completes the section on applying the identifier to a critical ocean acoustic estimation problem.

Table I. MBID: Wave Number Estimation

\begin{tabular}{||c|c|c||}
\hline \multicolumn{3}{|c|}{ Hudson Canyon Experiment Wave Numbers } \\
\hline Model & Simulation & Experiment \\
\hline 0.2079 & $0.2105 \pm 0.0035$ & $0.2076 \pm 0.0043$ \\
\hline 0.1991 & $0.1993 \pm 0.0052$ & $0.1978 \pm 0.0036$ \\
\hline 01827 & $0.1846 \pm 0.0359$ & $0.1817 \pm 0.0251$ \\
\hline 0.1746 & $0.1770 \pm 0.0149$ & $0.1746 \pm 0.0098$ \\
\hline 0.1423 & $0.1466 \pm 0.0385$ & $0.1479 \pm 0.0288$ \\
\hline
\end{tabular}

Table II. MBID: Modal Estimation

\begin{tabular}{|}
\hline \multicolumn{3}{|c|}{ Hudson Canyon Experiment: Modeling Error } \\
\hline Mode & Fixed MBP & Adaptive \\
\hline 1 & $1.8 \times 10^{-3}$ & $1.2 \times 10^{-3}$ \\
\hline 3 & $1.9 \times 10^{-1}$ & $3.0 \times 10^{-4}$ \\
\hline 4 & $5.8 \times 10^{-4}$ & $3.2 \times 10^{-4}$ \\
\hline 5 & $5.4 \times 10^{-4}$ & $6.7 \times 10^{-4}$ \\
\hline
\end{tabular}

\section{DISCUSSION}

In this paper we have developed an on-line, adaptive, model-based solution to the ocean acoustic signal processing problem based on coupling the normal-mode propagation model to a vertical sensor array. The algorithm employed was the nonlinear extended Kalman filter identifier/parameter estimator. It was shown that the model-based identifier (MBID) could be designed to estimate the set of horizontal wave numbers from noisy Hudson Canyon experimental data yielding results better than those reported previously [8] in the sense that the estimated modal functions "track" those predicted by propagation models more closely (smaller variances, etc.). It appears that the application of the adaptive MBID scheme yields superior performance then a
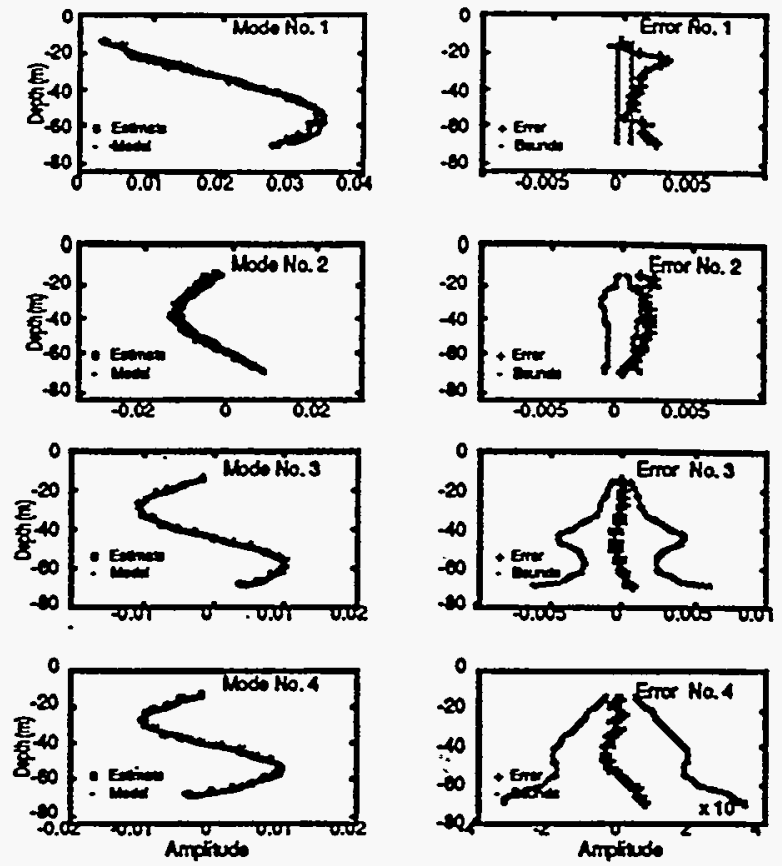

Figure 2: Model-Based Identification of Hudson Canyon Experiment (0.5Km): (a) Mode 1 and error ( $91 \%$ out). (b) Mode 2 and error ( $83 \%$ out). (c) Mode 3 and error (0\% out). (d) Mode 4 and error ( $0 \%$ out).
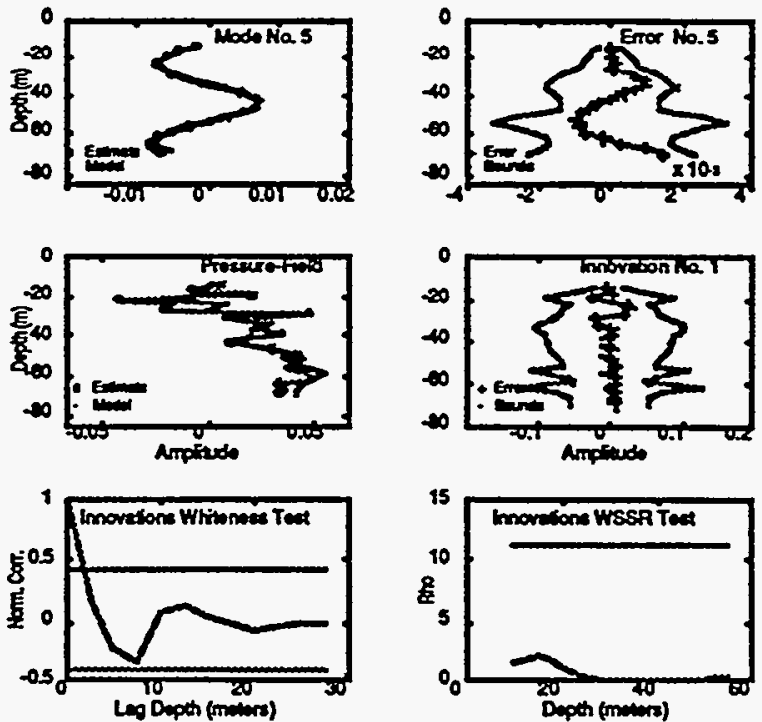

Figure 3: Model-Based Identification of Hudson Canyon Experiment (0.5Km): (a) Mode 5 and error ( $0 \%$ out). (b) Pressure-field and innovation (2\% - $0 \%$ out). (c) Whiteness test and WSSR ( $0 \%$ out). 

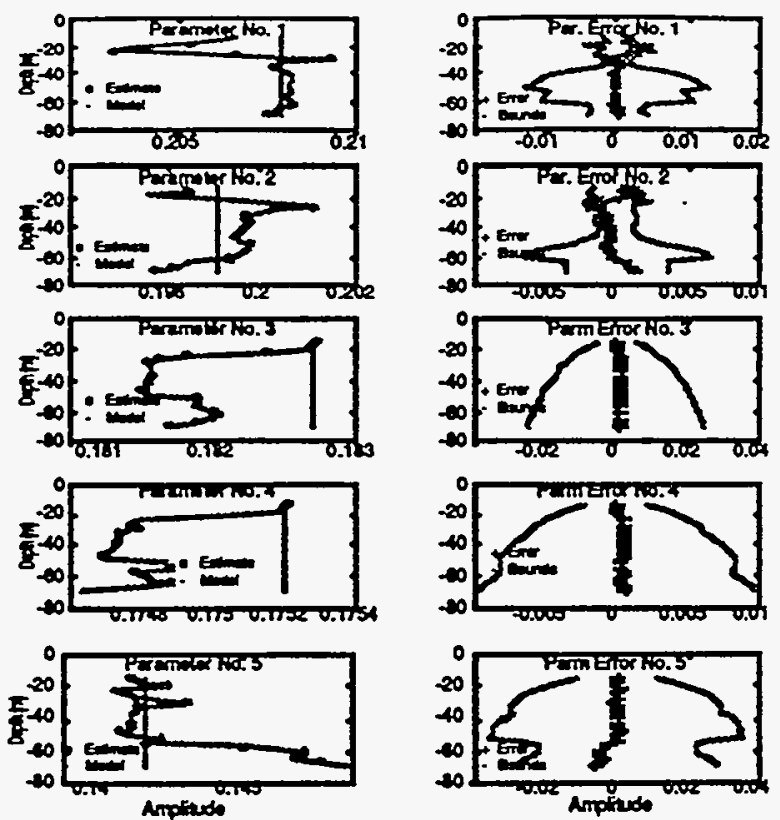

Figure 4: Model-Based Identification of Hudson Canyon Experiment (0.5Km): (a)Parameter 1 and error (8.7\% out). (b) Parameter 2 and error (4.4\% out). (c) Parameter 3 and error (0\% out). (d) Parameter 4 and error ( $0 \%$ out).

fixed processor enabling it to passively "listen" to the everchanging ocean environment and "learn" about its changes in the true spirit of a wellinformed array.

\section{References}

[1] E.J. Sullivan and D. Middleton, "Estimation and detection issues in matched-field processing", IEEE Trans. Ocean Eng., 18, (3), 156167,1993

[2] M. J. Hinich, "Maximum likelihood signal processing for a vertical array, J. Acoust. Soc. Am., 54, 499-503, 1973.

[3] H .P. Bucker, "Use of calculated sound fields and matched-field detection to locate sound in shallow water, "J. Acoust. Soc. Am., 59, 329-337, 1976.

[4] A. B. Baggeroer, W. A. Kuperman, and H. Schmidt, "Matched-field processing: source localization in correlated noise as an optimum parameter estimation problem, J. Acoust. Soc. $A m, 83,(2), 571-587,1988$.

[5] A. Jazwinski, Stochastic Processes and Filtering Theory. New York:Academic Press, 1970.
[6] J. V. Candy, Signal Processing: The Model Based Approach. New York:McGraw-Hill, 1986.

[7] J.V. Candy and E.J. Sullivan. "Model-based processor design for a shallow water ocean acoustic experiment ${ }^{n}$, J. Acoust. Soc. Am., 95, (4) 2038-2051, 1994.

[8] L. J. Ljung, System Identification: Theory for the User. New Jersey: Prentice-Hall, 1987.

[9] T. Soderstrom and P. Stoica, System Identification. New Jersey: Prentice-Hall, 1989.

[10] J.P. Norton, An Introduction to Identification. New York: Academic Press, 1986.

[11] J. V. Candy and E. J. Sullivan. "Ocean acoustic signal processing: a model-based approach." J. Acoust. Soc. Am., 92, (12), 3185$3201,1992$.

[12] J. V. Candy and E.J. Sullivan. "Sound velocity profile estimation: a system theoretic approach", IEEE Trans. Ocean Eng., 18, (3), 240-252, 1993.

[13] J.V. Candy and E.J. Sullivan. "Modelbased inversion for a shallow ocean application", Proc. Full-Field Inversion Confr., SACLANT, LaSpezia, Italy also J. Acoust. Soc. Am., to appear Oct.1995.

[14] C. S. Clay, and H. Medwin, Acoustical Oceanography. New York, Wiley, 1977.

[15] J. V. Candy, and E.J. Sullivan, "Passive localization in ocean acoustics: a model-based approach," LLNL Report, UCRL-JC-118546, also J. Acoust. Soc. Am., to appear Oct.1995.

[16] L. Ljung, "Asymptotic behavior of the extended Kalman filter as a parameter estimator for linear systems, IEEE Trans. Auto. Control, AC-24, 36-50, 1979.

[17] J.V. Candy and P. M. Candy, "SSPACK.PC: A model-based signal processing package on personal computers," DSP Applic., 2,(3), 3342, 1993.

[18] W. Carey, J. Doutt and L. Maiocco, "Shallow water transmission measurements taken on the New Jersey continental shelf, ${ }^{\prime} J$. Acoust. Soc. Am., 89, 1981(A), 1991.

\section{Acknowledgements}

This work received partial support from the Office of the Chief of Naval Research, OCNR N00014-92-F-0055 and the Lawrence Livermore National Labaratory under the auspices of the U. S. Department of Energy, DOE contract No. W7405-ENG-48. We would like to acknowledge the motivation and support of $\mathrm{Dr}$. R. Doolittle, Dr. D. Johnson and Cmdr. M. Shipley of OCNR and we would like to thank Dr. William Carey of of the Advanced Research Projects Agency for the use of the Hudson Canyon experimental data. 